\title{
Evolutionary Game Simulation: A Case In Water Resources Management
}

\author{
Xianshi $\mathrm{Li}^{1,2}$ \\ 1. Beijing Institute of Technology, Beijing 100081; 2. Hebei GEO University, Shijiazhuang 050031, China
}

Keywords: Simulation; evolutionary game; water resource; stakeholder.

\begin{abstract}
In this paper it simulates the dynamic evolution of water ecosystem by the evolutionary game theory. Through analyzing the theoretical hypothesis and model simulation, it finds that strategic choices of players are decided by payoffs of actions when water quality under certain conditions, there exist stable evolutionary equilibrium between water quality and players mixed strategy when payoffs of player' actions are exogenous variables. By simulating combined system dynamics in water resources, it finds that government can choose appropriate taxes and subsidies according to different water quality state if resource of river basin ecology has the ability to regenerate, so that stakeholders have to change their strategic choices, which make the combined system evolve to the optimal equilibrium..
\end{abstract}

\section{Introduction}

With the development of economy and the shortage of water resources, the conflict of water resources in China becomes more and more serious. The water resources governance has become a problem that scholars pay attention to. Because the public goods characteristics of water resources, it is easy to produce the "tragedy of the commons". From the point of water resources governance, it is mainly through reasonable institutional arrangements and coordination to maximize the total profits of water resources.

Because of the systematic nature of water ecology, people's different behavior strategies will bring different degrees of impact on water ecology in the river basin. In addition, the water resource itself has a certain degree of self purification capacity, reasonable pollution emissions will be purged, and excessive emissions will make the water resources irreversible. Therefore, in the water ecosystem, we must analyze the behavior strategies of the stakeholders on the water resources, and consider the different water resources regeneration ability and the different choices for the stakeholders. How to through the appropriate institutional arrangements and the rational use of water resources to achieve a dynamic balance between the water ecological systems is not the key to maintain the sustainable utilization of water resources, but also the implementation of effective management of water resources.
The evolutionary game model ${ }^{[1]}$ is combined with the game analysis and the dynamic evolution process. It emphasizes that the participants who need not have complete information and perfect rationality, which can reflect the evolution of population size and strategy. This game model is more convenient and efficient than the traditional model in the analysis of the game process. Therefore, this paper uses evolutionary game model to simulate the evolution of water resources system.

\section{Literature review}

Evolutionary game theory has been widely used in economics and social science research. In recent years, many scholars have applied evolutionary game theory to water resources and ecological field. Roseta-Palma ${ }^{[2]}$, Aggarwal ${ }^{[3]}$ Gaudet $^{[4]}$, Osés ${ }^{[5]}$ have constructed a dynamic model of the development and use of water resources, and they simulated the stable equilibrium conditions. Shahi ${ }^{[6]}$ use evolutionary game theory to analyze the forest joint management; they simulate the evolutionary equilibrium strategy and get the stable equilibrium state of multi agent evolutionary game. Blanco ${ }^{[7]}$ use evolutionary game model to analyze the complex system dynamics of eco tourism companies developing and utilizing shared resources, and it considers that there is a stable mixed equilibrium between natural resources and tourism company's unilateral commitment strategy. Luo Hui ${ }^{[8]}$ takes Shaanbei Loess Plateau as an example to apply static and evolutionary game theory method to draw the conclusion that it is necessity of government regulation in sustainable development of water resources. Chen Zhisong ${ }^{[9]}$ applies the evolutionary game theory to water resources allocation in river basin, and separately analyzes replicator dynamics and its evolutionary stable strategy among the water resources manufacturers and between the water resources manufacturers and the water resources administration. Cao Honghua ${ }^{[1]}$ uses evolutionary game model to study the ecological compensation and its stability strategy, and he stimulates the replicator dynamics, evolutionary stable strategy and system stability among different interest groups. Li Changfeng ${ }^{[10]}$ takes the Taihu River Basin as an example to study the basin ecological compensation by using the evolutionary game theory. Niu Wenjuan $^{[11]}$ uses evolutionary game model to simulate three scenarios in transboundary water conflict.

In these above studies, most of them study from the perspective of water resources or ecological resources users and government. It does not consider the evolutionary game path of the human behavior impact on water quality, and the 
impact of water resources on the choice of stakeholders' strategies, which regards the water resources system as a whole. Of course, the evolutionary game model constructed on these papers provides important ideas and references. In this paper, stakeholder' strategies and water resources are considered as a combined system, and it uses the evolutionary game model to simulate the evolution path between the stakeholder behavior choice and water resources system.

\section{Modeling and simulation}

\subsection{Model assumptions and construction}

First, it assumes that water users are homogeneous, that is, people's demands for water quality are consistent. Second, it assumes that the game participant' goal is the maximization of individual interests; the interests include economic interests and water environment interests. Third, it assumes that the water pollution is taxed and the government has a strict supervision.

The model consists of $\mathrm{N}$ participants to share the water resource ( $N=\{1, \ldots, n\}, n \geq 2)$. These participants are enterprises or residents, and they share the water resources. We set participant $\mathrm{I} \in \mathrm{N}, a_{i} \in\left\{a_{n g}, a_{g}\right\}$. Here $a_{g}$ means participant takes the "green" behavior and does not have a negative impact on water quality; $a_{n g}$ means that the participants do not adopt "green" behavior, so it has a negative impact on water quality. $n_{g}$ and $n_{n g}$ represent the proportion of "green" and "non green" behavior separately, so $n_{g}+n_{n g}=1$.

It is assumed that the costs of participants who choose green behavior and non green behavior are $c\left(a_{g}\right)$ and $c\left(a_{n g}\right)$, and $c\left(a_{g}\right)>c\left(a_{n g}\right)$. To simplify the analysis, it assumes that $c\left(a_{n g}\right)=0$, then the profits of participants who choose the green behavior

are $\pi_{g}=y(\Psi)-c\left(a_{g}\right)$

Here, $\Psi$ is the quality and function $y$ is of water quality; participants' profits are different under different water quality, and $y^{\prime}(\Psi)>0 \quad y^{\prime \prime}(\Psi)<0$.

Participants' profits who choose non green behaviors are $\pi_{n g}=(1-t) y(\Psi)$

Here, $\mathrm{t}$ is the tax rate in accordance with the income. The participant' strategies depended on extra cost $c\left(a_{g}\right)$ and non green behavior income loss $t y(\Psi)$. According to evolutionary game theory, the difference of participant payment impact on the proportion $n_{g}$ and $n_{n g}$. Dynamic characteristics of evolutionary stable strategies are obtained by using replicator dynamic equation: $\dot{n}_{g}=n_{g}\left(\pi_{g}-\bar{\pi}_{g, n g}\right)$

Here, $\bar{\pi}_{g, n g}$ is the average profit of all participants, $\bar{\pi}_{g, n g}=n_{n g} \pi_{n g}+n_{g} \pi_{g}$. From formula (1) and formula (2) and formula (3),we will get the replicated dynamic equation, $\dot{n}_{g}=n_{g} n_{n g}\left(t y(\Psi)-c\left(a_{g}\right)\right)$
It is assumed that the water quality function is as follows: $\dot{\Psi}=f(\Psi)-D\left(n_{n g}\right)$

Here, $f(\Psi)$ is the reproductive function of water resources, $D\left(n_{n g}\right)$ is total pollution that the participants choose the mixed behavior strategies on the water resources. It is assumed that the maximum carrying capacity of water quality and the minimum carrying capacity is $\underline{\Psi}$ and $\bar{\Psi}$, and $f(\underline{\Psi})=0, f(\bar{\Psi})=0,(0<\underline{\Psi}<\bar{\Psi})$, which means that the water recycling capacity is zero beyond the water resources carrying level. In the interval range $(\bar{\Psi}, \underline{\Psi}), f(\Psi)>0$, $f^{\prime}(\Psi)<0$, that is, water resources make water better through regeneration, but the ability to regenerate gradually declined. At this time, the relationship between water quality and water regeneration capacity is inverted $U$ shape curve. When $\underline{\Psi}<\Psi<\Psi_{\mathrm{M}}$, the water regeneration capacity is increasing; when the water quality is $\Psi=\Psi_{\mathrm{M}}$, the water regeneration capacity reached the peak; when $\Psi_{M}<\Psi<\bar{\Psi}$, the water regeneration capacity is declining.

The pollution function $D\left(n_{n g}\right)$ for the mixed behavior of the participants, we assume that participants' green behaviors do not have a negative impact on water resources, and non green behaviors' pollution is $d$. The total negative impacts of participants on the environment are $D\left(n_{n g}\right)=N d n_{n g}$.

\subsection{Dynamic evolution of participants' strategies}

It assumes that the water quality is certain; the dynamic of participant behavior is formula (4). The system is in steady state $\dot{n}_{g}=0$, there are three stable states at this time: first, it is $t<c\left(a_{g}\right) / y(\Psi), n_{g}=0$, and all participants choose pure strategy $a_{n g}$; second, it is $t>c\left(a_{g}\right) / y(\Psi), n_{g}=1$, and all participants choose pure strategy $a_{g}$; third, it is $t=c\left(a_{g}\right) / y(\Psi), 0<n_{g}<1$, and participants select mixed strategies.

From the view of sustainable utilization and governance of water resources, the pure strategy $a_{g}$ is the most ideal result in the three game models. Mixed strategy is also a better result than pure strategy $a_{n g}$ when the participants don't choose the green action. Therefore, under the condition of cost and income that the participant chooses the green behavior is certain; the government can influence the participants' strategies through the tax in order to achieve the ideal stable condition. For the fixed water quality conditions, the evolution equilibrium of participant behavior is determined by the ratio of green behavior cost $c\left(a_{g}\right)$ and pollution tax rate t. Raising the water pollution tax rate will make steady state to the government expect direction. Similarly, under the condition of certain pollution tax rate, the government can also adopt various subsidy policies to change the participants' green behavior cost to influence the behaviors. That is, when $c\left(a_{g}\right)>\operatorname{ty}(\Psi)$, it is $n_{g}=0$, all participants choose pure strategy $a_{n g}$; when $c\left(a_{g}\right)<t y(\Psi)$, it is $n_{g}=1$, all participants choose pure strategy $a_{g}$; when $c\left(a_{g}\right)=\operatorname{ty}(\Psi)$, it is $0<n_{g}<1$, the participants choose mixed strategies. Therefore, in addition to taxation, the government can take various subsidies to reduce the green 
behavior cost, so that the equilibrium moves to the desired direction.

\subsection{Dynamic evolution of water quality}

It assumes that the participants' strategies are exogenous, and we simulate the dynamic change of water quality. According to formula (5), it can get the stable condition $D\left(n_{n g}\right)=f(\Psi)$. For $D\left(n_{n g}\right)=N d n_{n g}$, it can get the relationship between strategies and water quality, $f(\Psi)=N d n_{n g}$. because of the exogenous variables $N$ and $d$, we get the curve of $\Psi$ and $n_{n g}$, as shown in Figure 1. $\tilde{\Psi}\left(n_{n g}\right)$ and $\hat{\Psi}\left(n_{n g}\right)$ is the relationship of $\Psi$ and $n_{n g}$, under the condition of $\dot{\Psi}=0$. At any point of the two curves, it meets $D\left(n_{n g}\right)=f(\Psi)$. Due to the fixed strategies, we can simulate the water quality trends based on different water quality conditions. As shown in Figure 1 of region A, the water quality is lower than $\tilde{\Psi}(0)$, and the water quality will reduce whatever participants take any action. In the area $\mathrm{B}$, although the water resources have the self-purification capacity, the water quality is declining because the pollution degree is greater than the regeneration capacity of water resources. In the area $\mathrm{C}$, the water quality is improving for the water resources regeneration capacity greater than the negative impact caused by pollution. In area $D$, the regeneration capacity of water resources is less than the negative impact, so it decreases the water quality. Therefore, the water quality converges to the right curve $\hat{\Psi}\left(n_{n g}\right)$. $\hat{\Psi}\left(n_{n g}\right)$ and $\tilde{\Psi}\left(n_{n g}\right)$ simulate the corresponding relationship curves of $\Psi$ and $n_{n g}$ under the equilibrium conditions of water quality. In the right curve, $\hat{\Psi}\left(n_{n g}\right)$ means that high water quality is a stable equilibrium and it is the reverse direction change of $\Psi$ and $n_{n g}$. In the left curve, $\tilde{\Psi}\left(n_{n g}\right)$ is a unstable equilibrium, and with the same direction change of $\Psi$ and $n_{n g}$.

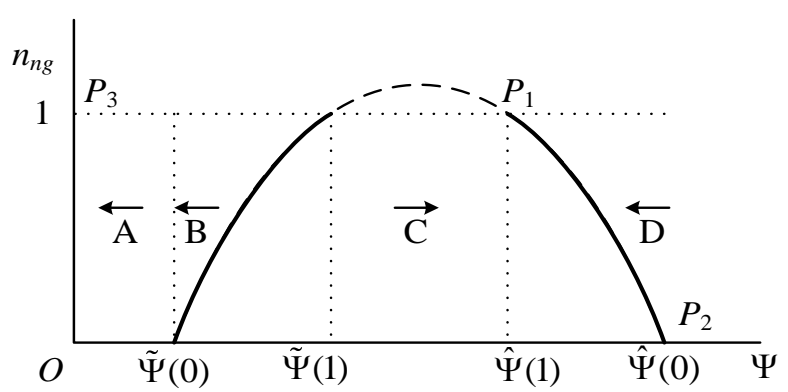

Figure 1 The dynamic model of water quality evolution

\subsection{Dynamic evolution of water combined system}

In the water complex system involved water quality and participation strategies, $\Psi$ and $n_{n g}$ are endogenous variables. The dynamic evolution of complex system depends on formula (4) and formula (5). Under the condition of system stability, water quality meets two conditions: $\dot{\Psi}=0$ and $\dot{n}_{g}=0$. Because of the different water conditions and different benefits, the evolutionary stable strategies are depend on $\mathrm{c}\left(\mathrm{a}_{\mathrm{g}}\right)$ and $t$ and $\Psi$. Under the condition of water quality stability, the relationship between water quality and non green action is determined by the water regeneration ability and the pollution of participants. We analyze the dynamic evolution of water ecosystem by 5 different intervals.

( I ) If $\Psi<\tilde{\Psi}(0)$, water quality will continue to decline regardless of the strategies.

(II) If $\tilde{\Psi}(0)<\Psi<\tilde{\Psi}(1)$, there are two evolution path. If $\mathrm{c}\left(\mathrm{a}_{\mathrm{g}}\right) / \mathrm{t}<\mathrm{y}(\Psi), \mathrm{n}_{\mathrm{g}}=1$, all participants choose the pure strategies $\mathrm{a}_{\mathrm{g}}, \mathrm{D}\left(\mathrm{n}_{\mathrm{ng}}\right)=0$ means that the water quality will continuous improve for no pollution of water resources. If $c\left(a_{g}\right) / t>y(\Psi)$, $\mathrm{n}_{\mathrm{g}}=0$, all participants choose pure strategy $\mathrm{a}_{\mathrm{ng}}, \mathrm{D}\left(\mathrm{n}_{\mathrm{ng}}\right)=\mathrm{Ndn}_{\mathrm{ng}}$, the water quality will deteriorate for the negative impact of pollution more than the regeneration ability.

(III)If $\tilde{\Psi}(1)<\Psi<\hat{\Psi}(1)$, the water quality will certainly improve no matter what people choose the strategies, because the negative impact of water resources regeneration ability is always greater than the water pollution. Especially, if $\mathrm{c}\left(\mathrm{a}_{\mathrm{g}}\right) / \mathrm{t}<\mathrm{y}(\Psi)$, the water quality will improve faster for there is no pollution.

(IV) If $\hat{\Psi}(1)_{<\Psi<} \hat{\Psi}(0)$, there are two evolution path too. if $c\left(a_{g}\right) / t<y(\Psi)$, all participants select the pure strategy $a_{g}$, $\mathrm{D}\left(\mathrm{n}_{\mathrm{ng}}\right)=0$, so the water quality is $\hat{\Psi}(0)$. If $\mathrm{c}\left(\mathrm{a}_{\mathrm{g}}\right) / \mathrm{t}>\mathrm{y}(\Psi)$, all participants have pure strategies $a_{n g}, D\left(n_{n g}\right)=N_{d n}$, the water resources regeneration ability is lower than the negative effects caused by water pollution, so the water quality is $\hat{\Psi}(1)$.

(V)If $\Psi>\hat{\Psi}(0)$, the water quality reaches to an extreme state regardless of what people choose the strategies, and the water quality will converge to the $\hat{\Psi}(1)$.

Therefore, there are three stable equilibrium points $P_{1}(\hat{\Psi}(1), 1), P_{2}(\hat{\Psi}(0), 0)$ and $P_{3}(0,1)$ in water ecosystem, and other points except $\mathrm{P} 1$ and $\mathrm{P} 2$ are unstable equilibria in curve $\hat{\Psi}\left(n_{n g}\right)$ and $\tilde{\Psi}\left(n_{n g}\right)$. Equilibrium point P1 satisfies condition $y(\hat{\Psi}(1))<c\left(a_{g}\right) / t$; equilibrium point P2 satisfies condition $y(\hat{\Psi}(0))>c\left(a_{g}\right) / t$.

When the water quality is $\Psi \in\left(\tilde{\Psi}(0), \hat{\Psi}_{(0)) \text {, water resources }}\right.$ has regenerative capacity, the government only needs to adjust the rate of pollution tax and subsidy policies to affect the cost of green and non green behavior, so $c\left(a_{g}\right) / t<y(\Psi)$. All participants will choose pure strategy $a_{g}$ to improve the water quality. After the water quality has been improved, the complex system states are being a good stable state $\hat{\Psi}(1)$ and the best stability $\hat{\Psi}(0)$, which are corresponding to the two kinds of pure strategies $n_{n g}=1$ and $n_{n g}=0$. However, if the governmental policies are failure to make $c\left(a_{g}\right) / t>y(\Psi)$, and 
the water quality status at this time is lower than $\tilde{\Psi}(1)$, the water quality will inevitably lead to the continued deterioration because the water resources regeneration capacity is less than the pollution. The ultimately evolution equilibrium point of the complex system is $P_{3}$. In addition to the water quality below the state $\tilde{\Psi}(0)$, it is necessary for the government to take coercive interventions against water pollution for it is the most extreme conditions. The government can use various tax and subsidy policies to keep equilibrium optimal evolution of water ecosystem according to the water quality in different states.

It should be noted that in real life, equilibrium point $\mathrm{P}_{1}$ may not exist. Because if there are enough participants in the game, and $d$ is large enough to meet the conditions of $N d n_{n g}>f\left(\Psi_{\mathrm{M}}\right)$, $P_{1}$ does not exist. At this point, the water ecosystem has only stable equilibrium point $P_{2}(\hat{\Psi}(0), 0)$ and $P_{3}$. In order to realize the water ecosystem to reach the equilibrium point of $P_{2}$, we must ensure that the water quality always meets the $c\left(a_{g}\right) / t<y(\Psi)$, so the participants choose the pure strategies $a_{g}$. Otherwise, once the participants choose pure strategy $a_{n g}$, water quality will continue to decline to the state of $\Psi<\tilde{\Psi}(0)$, water ecosystem can not be achieved the regeneration ability.

\section{Summary}

In this paper, evolutionary game model is used to simulate the dynamic evolution process of water resources stakeholder's behaviors and water ecosystem. Through the theoretical hypothesis and modeling simulation, we found that in certain water quality conditions, the participants choose the strategies depend on different behaviors of payments; when the strategies are exogenous variables, and the water quality in high state, there is stability of evolutionary equilibrium between water quality and the mixed strategies. Based on the water ecosystem evolution simulation, we found that when water resources have regenerative capacity, the government needs to ensure the "green" payments always greater than the "non-green" behaviors; the water ecosystem will naturally evolve to the optimal equilibrium level.

Of course, the model also has some strict assumptions. For example, participants are homogeneous. In real life stakeholders may have different demands. In addition, the costs and benefits of various strategies are not necessarily consistent. The government supervision for the water pollution and pollution tax is not strict, and in many cases there is lack of supervision. Moreover, the pollution of water resources in the transboundary basin is more complex, because of different administrative powers and governance objectives; it will inevitably affect the evolution path of complex ecosystems. The transfer of benefits between different groups and upstream and downstream is not reflected in this paper, which will also affect the evolution of the ecosystem. These need further in-depth research in the future.

\section{Acknowledgements}

This paper is supported by the social science foundation of Hebei province (No. HB16YJ021).

\section{References}

[1]Cao Hong-hua,Jing Peng,Wang Rongcheng. “The Dynamic Evolution Mechanism of Ecological Compensation and Its Stable Strategy Research",Journal of natural resources. 9,pp.1547-1555,(2013).

[2]Roseta-Palma,C. "Ground water Management When Water Quality is Endogenous",Journal of Environmental and Management. 44,pp. 93-105,(2002).

[3]Aggarwal, R. M., Narayan, T.A. "Does Inequality Lead to Greater Efficiency in the Use of Local Commons? The Role of Strategic Investment in Capacity". Journal of Environmental and Management. 47, pp.163-182, (2004).

[4]Gaudet, G., Moreaux, M., Withagen, C. "The Alberta Dilemma: Optimal Sharing of a Water Resource by an Agricultural and Oil Sector", Journal of Environmental and Management. 52, pp. 548-566, (2006).

[5]Osés, N., Viladrich, M. "On the Sustainability of Common Property Resources ",Journal of Environmental Economics and Management, 53,pp.393-410. (2007).

[6]Shahi, C., Kant, S. "An Evolutionary Game-theoretic Approach to the Strategies of Community Members under Joint Forest Management Regime".Forest Policy and Economics,9, pp.763-775.(2007).

[7]Blanco, E., Lozano, J., Rey-Maquieira, J. “A Dynamic Approach to Voluntary Environmental Contributions in Tourism”, Ecological Economics, 69,pp.104-114.(2010),

[8] Luo Hui,et al. "Functions of Government Regulation in Sustainable Development of Water Resources". Bulletin of Soil and Water Conservation. 5,pp.94-99.(2005)

[9]Chen Zhisong,et al. "Evolutionary Game Analysis of Water Resources Allocation in River Basin", Chinese Journal of Management Science, 6,pp.176-183.(2008).

[10]LI Changfeng,et al. "Research on Basin Ecological Compensation Based on Evolutionary Game Theory", China Population Resources and Environment, 24,pp.171-176.(2014) [11]Niu Wen-juan,Wang Hui-min,Niu Fu. "Evolutionary Game Analysis on the Local Protectionism Behavior of Trans-boundary Water Conflict", Journal of Industrial Engineering/Engineering Management, 2,pp.64-72.(2014). 